\title{
spécification des charges et des écoulements dans les silos métalliques
}

\author{
specification of loads and flow patterns in steel silos
}

\author{
A. KHELIL, J.-C. ROTH \\ LEMTA UA 875 CNRS/GRRS IUT GC Le Montet*
}

Rev. Franç. Géotech. n 52 , pp. 11-25 (juillet 1990)

\section{Résumé}

Cet article présente une partie des travaux effectués dans le cadre de la " Mission Silos " en collaboration avec le CTICM.

Deux aspects nous ont intéressés : d'abord l'analyse des différents types d'écoulement durant la vidange caractérisés par la détermination des profils de vitesse sur modèle réduit ; ensuite l'interprétation des résultats des mesures expérimentales des efforts en paroi comparés aux méthodes de calculs de WALTERS, SOKOL et à la norme DIN.

Cette analyse comparative démontre que les modèles de calcul établis dans le cas d'un écoulement en masse ne peuvent pas être transposés aux cas de silos à vidange en cheminée.

\footnotetext{
Abstract

A part of the work developed with the CTICM for the "Mission Silo " is revealed in the paper.

This part regroups two main subjects. On the one hand, the different flow patterns which appear during discharge are analysed by the determination of the velocity profile in small-scale model silo. On the other hand, the comparison between experimental results of the wall pressure and the calculus wiht Walter's method, SOKOL's method and the norm DIN, is developed. This analysis shows that all the theoretical methods related to the mass flow are not verified for the funnel flow silo.
} 


\section{INTRODUCTION}

Nous nous proposons dans cet article de caractériser les profils de vitesse et les effets de pression et de frottement en paroi dans le cas du remplissage et de la vidange des silos métalliques.

Cette étude effectuée dans le cadre de la "Mission Silos» du Ministère de la Recherche et de la Technologie s'appuie sur les résultats expérimentaux obtenus :

- sur la base expérimentale de silos métalliques de Chartres dans le cas de la cellule " $D$ * de section circulaire ;

- en laboratoire à l'aide d'un silo modèle à l'échelle $1 / 20$ de la cellule « $A$ * de la base de Chartres.

L'objectif de ce travail est de participer à la mise au point d'un modèle théorique de calcul des pressions afin de définir une norme française pour la conception des silos métalliques.

Il existe depuis les travaux de ROBERT en 1892, qui le premier a montré que la poussée totale sur le fond d'un silo était inférieure au poids du matériau contenu dans le silo, de nombreux modèles analytiques de calcul des pressions. Ces méthodes ont évoluées dans le temps et peuvent être classées en deux grandes familles:

- les méthodes de calcul des pressions statiques : RANKINE (1857), JANSSEN (1895), KOENEN (1896), AIRY (1897), et REIMBERT (1953), basées sur l'équilibre statique de la matière ensilée à l'intérieur des cellules de stockage;

- les méthodes de calcul des pressions totales. CAQUOT (1956), THEIMER (1957), GENIEV (1958), PLATANOV et KOVTUN (1959), PEIPER et WENZEL (1962), WALKER (1966), JENIKE (1968), SAFARIAN (1969), WALTERS (1973), ENSTADT (1975), SOKOL (1984). Ces pressions totales (pressions latérales et verticales) prennent en compte les pressions statiques auxquelles on ajoute les surpressions en cours de vidange dues à des causes aussi variées que : modifications brutales du mode d'écoulement, de la vitesse d'écoulement, chocs dus aux ruptures de voutes, etc.

Dans la suite nous nous attacherons à prendre en compte, seulement les méthodes de WALTERS et de SOKOL qui à notre avis rendent bien compte des processus de remplissage et de vidange. Un bref rappel de ces deux techniques de calcul des pressions totales permettra de mettre en évidence les hypothèses indispensables à la mise en cuuvre de ces méthodes. Tous les modèles analytiques utilisés aujourd'hui pour le calcul des efforts de pression dans les silos découlent des équations de JANSSEN. Quelques modifications ou hypothèses supplémentaires ont été apportées par WALTERS et SOKOL.

WALTERS [15], considère une répartition non uniforme de la contrainte verticale sur une couche horizontale de matière ensilée en équilibre. Ainsi le coefficient de pression $\lambda$ est considéré comme le rapport de la contrainte horizontale s'exerçant à un certain niveau du silo sur la contrainte verticale moyenne. Le coefficient de pression $\lambda$ est fonction de langle de frottement interne et de l'angle de frottement en paroi. Suivant les valeurs de $\lambda$, WALTERS définit l'état de contraintes statiques associé au remplissage et l'état de contraintes dynamiques associé à la vidange.

SOKOL [14] poursuivant les travaux de FREESE [4] intègre dans les équations de JANSSEN la variabilité de $\lambda$ en fonction de la profondeur. La matière ensilée se trouve durant la vidange dans un état variable oscillant entre l'état d'équilibre au remplissage et l'état d'équilibre durant la vidange.

Ces méthodes de calcul basées sur l'équilibre d'une couche horizontale de matière ensilée, supposent que la matière glisse globalement comme une masse solide sur la paroi durant la vidange. Ceci implique que la vidange s'effectue en masse.

\section{MODE D'ÉCOULEMENT DANS LES SILOS. DÉTERMINATION DES PROFILS DE VITESSE}

La détermination expérimentale des profils de vitesses est effectuée sur modèle réduit à l'échelle $1 / 20$ de la cellule $\propto A$ A de la base de Chartres (cellule " $\mathrm{A}$ " de dimension : $17 \mathrm{~m}$ de hauteur et $6,42 \mathrm{~m}$ de côté). La géométrie de la cellule "A " permet des mesures aisées de vitesses. Son rayon hydraulique étant voisin de celui de la cellule « $D$ », seule la hauteur d'ensilage peut influencer les types d'écoulement observés dans ces deux cellules.

La matière ensilée considérée est le blé utilisé dans les essais à Chartres. Le modèle réduit est muni d'une paroi vitrée à travers laquelle s'effectue la visualisation de l'écoulement. Les profils de vitesses obtenus sont portés sur un diagramme représentant une partie du silo modèle avec les différentes cotes $z$ allant de 0 à $45 \mathrm{~cm}$. L'origine des vitesses est à chaque fois déplacée vers la cote $z$ où le profil de vitesses est déterminé.

\subsection{Ecoulement en masse (fig. 1)}

Cet écoulement apparaît généralement dans les silos munis de trémies assez lisses et suffisamment inclinées. Dès le début de la vidange, toute la matière descend régulièrement et on observe un mouvement d'ensemble de la matière.

L'écoulement en masse se caractérise par une vitesse régulière et relativement faible. La figure 2 montre le profil de vitesses verticales obtenu sur le modèle réduit de la cellule " $\mathrm{A}$ ", à la cote $40 \mathrm{~cm}$. Ce profil de vitesses est assez uniforme et s'étend sur la presque totalité de la largeur du silo. 


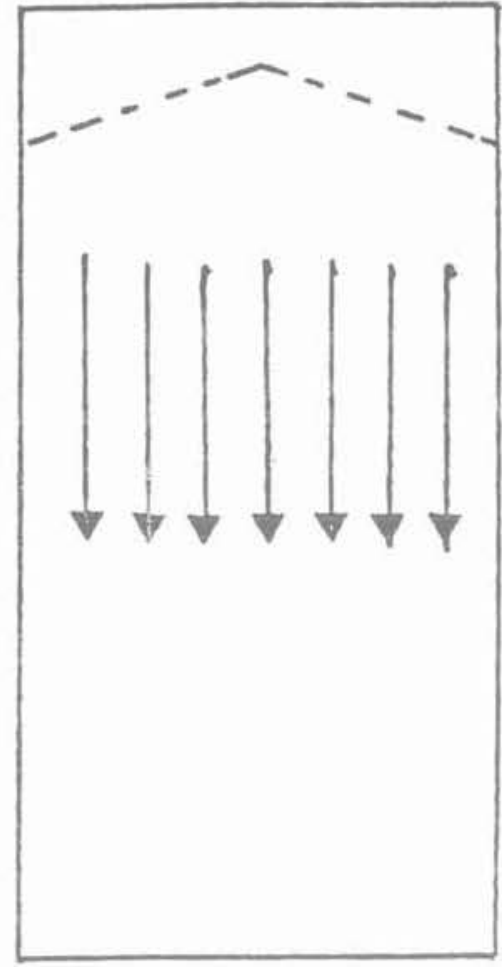

Fig. 1. - Ecoulement en masse. Fig. 1. - Mass flow.

\subsection{Ecoulement en cheminée (Funnel flow)}

Ce type d'écoulement apparait essentiellement dans les silos à fond plat ou avec trémies à faibles pentes. La matière s'écoule par une cheminée verticale (fig. 3). La cheminée est souvent de forme conique avec le diamètre inférieur ou approximativement égal à la dimension de l'orifice de vidange. De sérieux problèmes apparaissent pour les matières qui présentent de mauvaises propriétés d'écoulement). Avec le tassement, la matière augmente de consistance et reste immobile près des parois quand la zone active dans la cheminée est complètement évacuée, formant ainsi une sorte de tuyau.

L'écoulement en cheminée est souvent mal défini pour les matières cohérentes. Ainsi une partie de la matière entourant la cheminée reste instable et dans ces conditions, elle provoque un écoulement pulsatoire entraînant une sollicitation dynamique aléatoire de la structure du silo.

La figure 4 montre le profil de vitesses verticales obtenu à la cote $z=25 \mathrm{~cm}$, c'est-à-dire dans la partie basse du silo modèle. Sur cette figure on distingue bien la zone morte de part et d'autre du domaine d'écoulement caractérisé par le profil de vitesses.

\subsection{Ecoulement en semi-masse}

Ce troisième type d'écoulement est intermédiaire entre l'écoulement en masse et l'écoulement en cheminée. La figure 5 montre un silo à écoulement en semimasse. La partie basse fonctionne en écoulement en

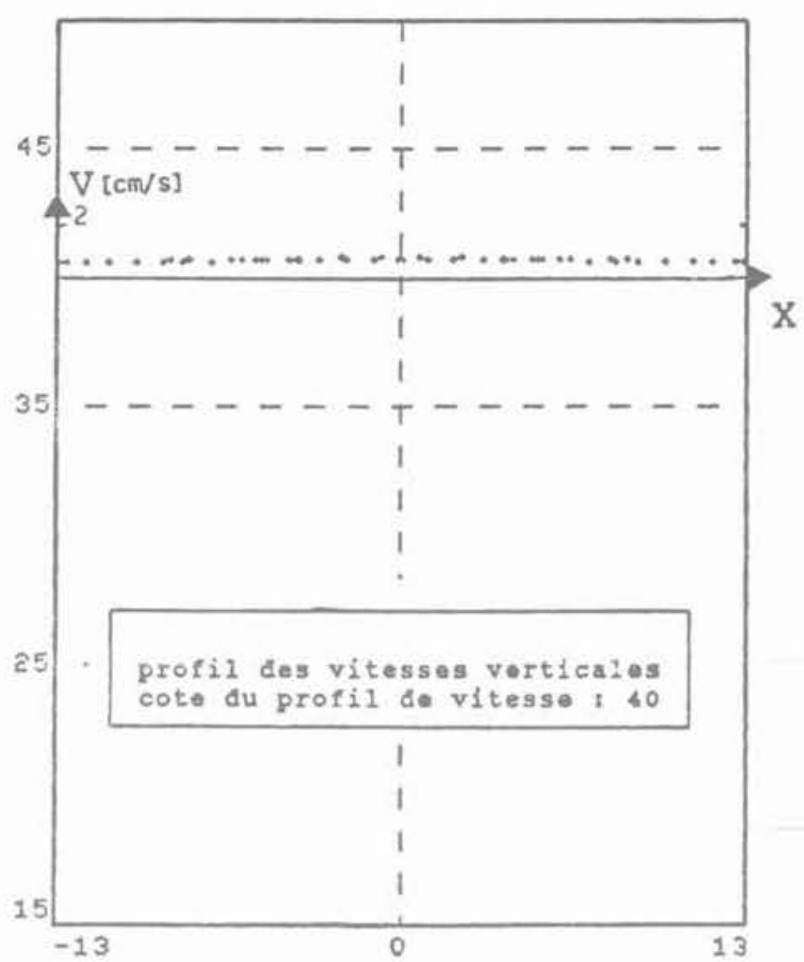

Fig. 2. - Profil de vitesses.

Fig. 2 - Velocity profile

cheminée, définissant une zone morte le long des parois et la partie haute fonctionne en écoulement en masse. Les profils de vitesses obtenus sur notre modèle réduit rassemblés sur un même diagramme

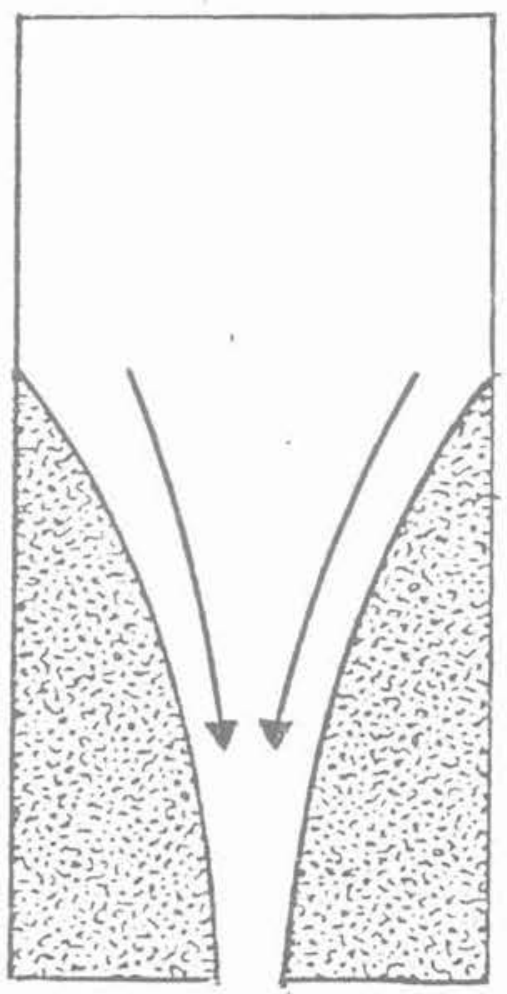

Fig. 3. - Ecoulement en cheminée. Fig. 3. - Funnel flow. 


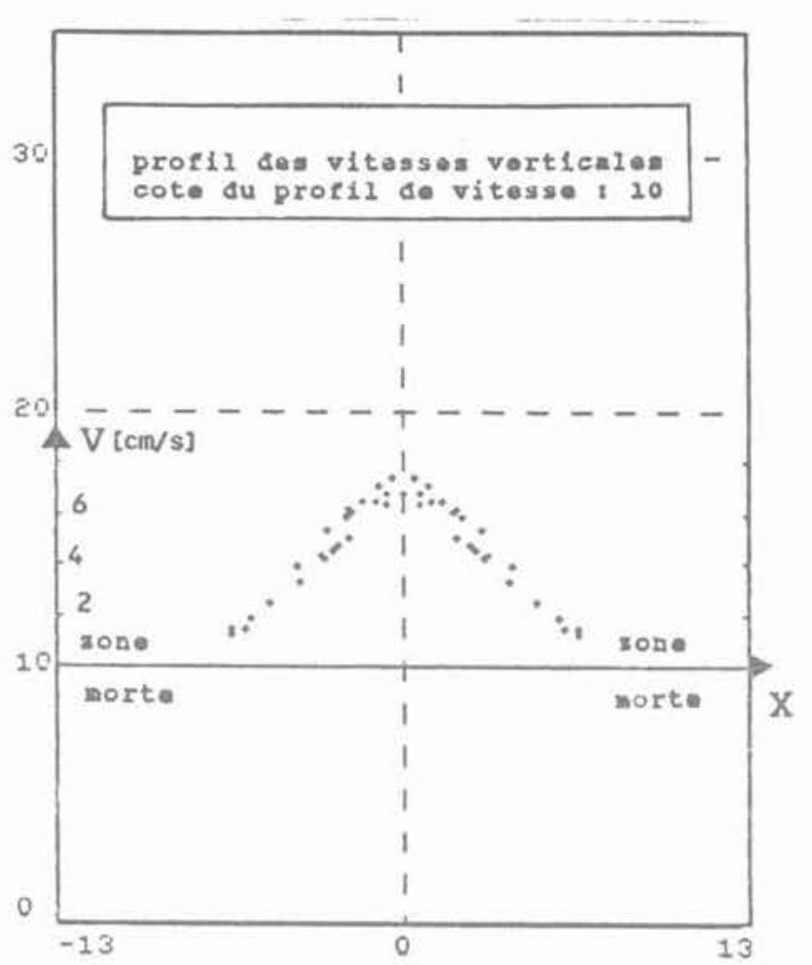

Fig. 4. - Profil de vitesses.

Fig. 4. - Velocity profile.

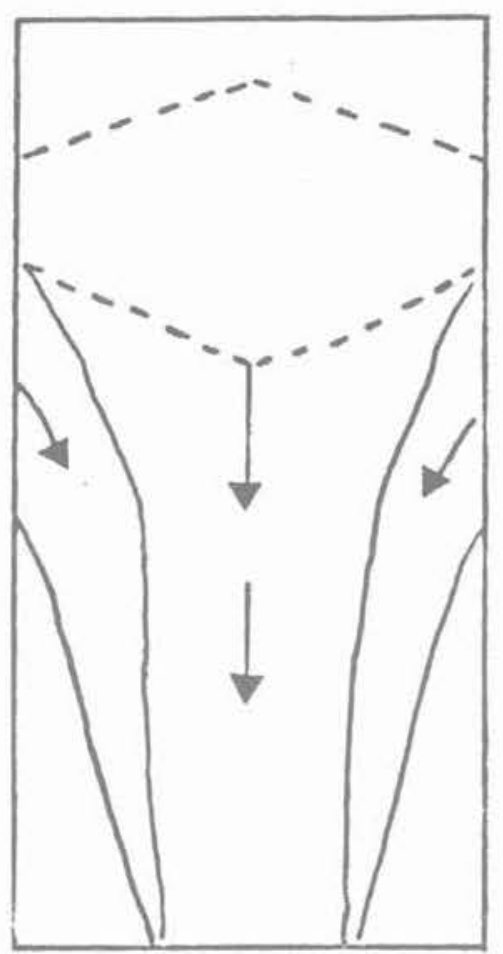

Fig. 5. - Ecoulement en semi-masse.

Fig. 5. - Semi-mass flow.

(fig. 6), dessinent les limites géométriques de la cheminée d'écoulement. La courbe limitant l'écoulement permet de distinguer la zone de transition entre l'écoulement en masse et l'écoulement en cheminée. Cette

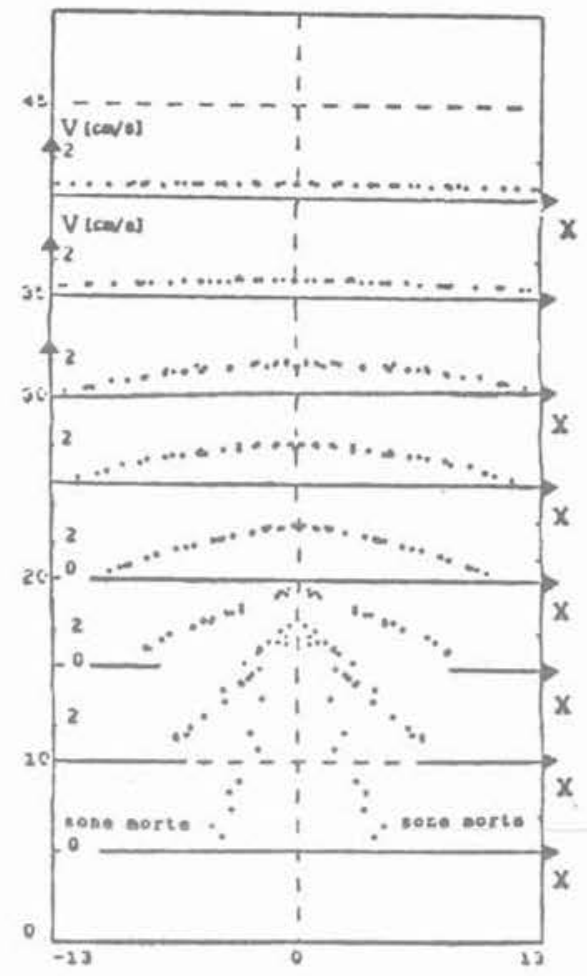

Fig. 6. - Profils de vitesses.

Fig. 6. - Velocity profiles.

zone de transition constitue un domaine probable de surpression.

Sur le modèle étudié, l'écoulement s'effectue globalement en semi-masse. Nous avons montré les profils de vitesses dans les parties de la cellule où l'écoulement s'effectue respectivement en masse et en cheminée.

Si la plupart des modèles de calcul des efforts de pression supposent l'écoulement en masse à la vidange pour respecter l'équilibre d'une couche horizontale de matière ensilée, il est important de signaler que ce type d'écoulement provoque de fortes pressions durant la vidange. L'avantage d'un silo à écoulement en masse est la facilité d'évacuation de la matière. Ainsi les problèmes de mises en voûte et de ségrégation sont évités.

\section{RÉSULTATS DE MESURES SUR SILOS EN VRAIE GRANDEUR A CHARTRES}

Nous présentons quelques résultats partiels obtenus sur la base expérimentale de Chartres pour mieux comprendre l'incidence du mode d'écoulement décrit précédemment sur les efforts en paroi.

\subsection{Variation des contraintes au cours des phases d'ensilage}

L'action de la matière ensilée sur les parois de la cellule est mesurée au moyen de capteurs spéciaux, 


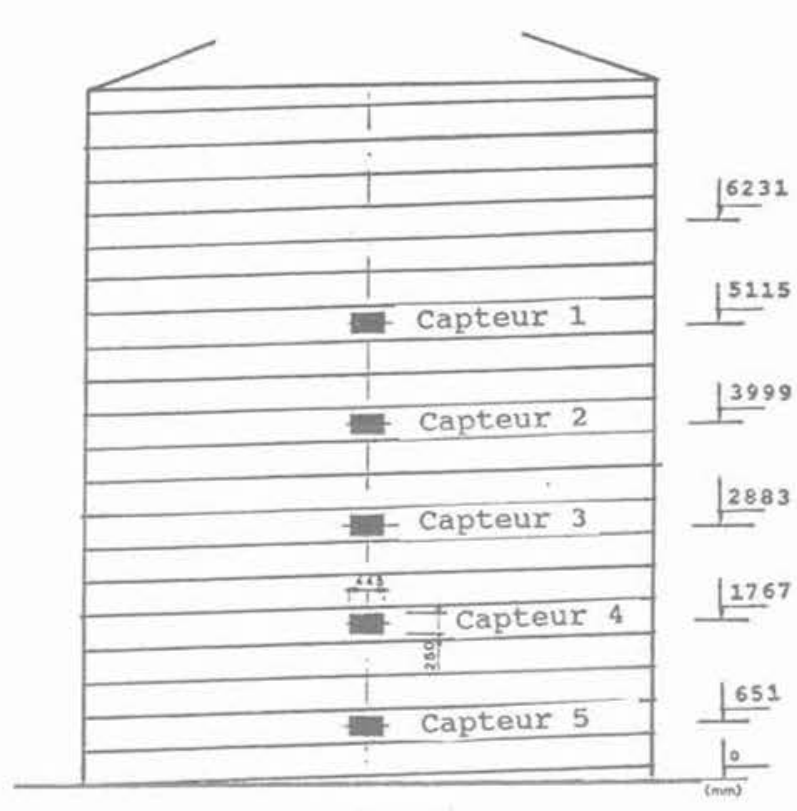

Gonerstrice 1

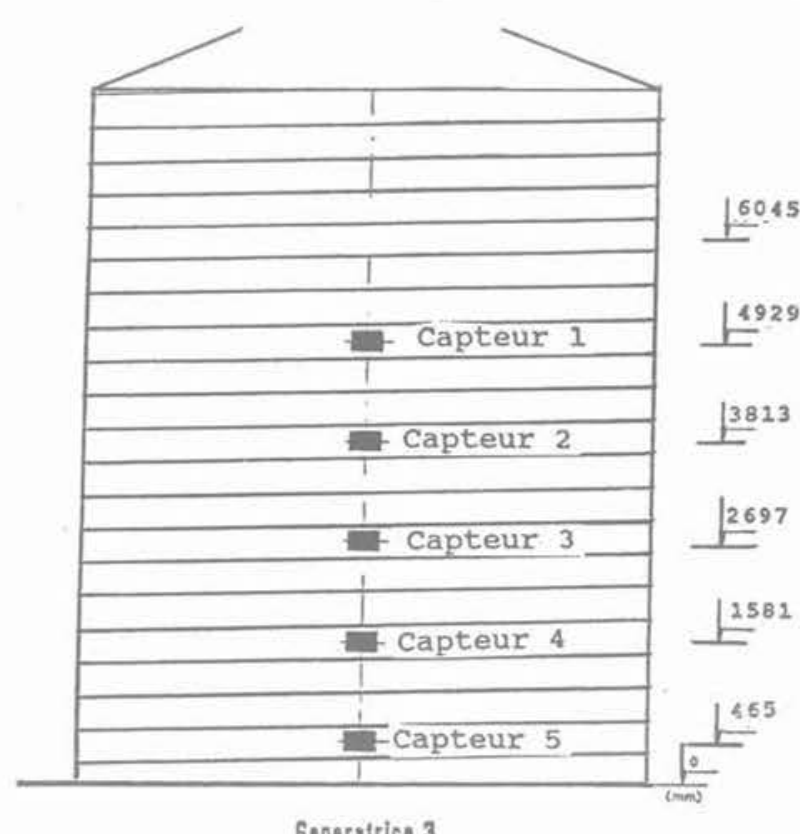

Goneratrice 3

Fig. 7. - Implantation des capteurs de parois. Fig. 7. - Position of wall transducers.

mesurant deux composantes de pression : la pression horizontale $\sigma x$ et le frottement $\tau$ [1]. La figure 7 décrit limplantation des capteurs de parois sur les génératrices principales 1 et 3 de la cellule * $D$ » exploitée sur le site de Chartres.

Cette cellule à fond plat avec un élancement 1,25 favorise l'écoulement en cheminée. En effet dès le début de la vidange on voit l'affaissement du cône d'ensilage qui par la suite évolue pour constituer une cheminée d'écoulement.

Les figures 8 et 9 représentent les variations de contraintes horizontales et de frottement obtenues au cours de la vidange pour les différents capteurs de paroi. Sur ces courbes on constate qu'au début de la vidange, les contraintes n'augmentent que légèrement par rapport à celles obtenues à la fin du remplissage. Cette augmentation se fait sentir différemment sur la hauteur de la cellule. Le capteur 5 situé dans la partie basse du silo (niveau $0,651 \mathrm{~m}$ ), indique une très faible augmentation.

Ces courbes (fig. 8 et 9) indiquent approximativement les zones où la matière en vidange est en mouvement, ou immobile. En effet les capteurs du haut de la cellule (capteur 1, 2, 3), indiquent une augmentation plus significative des contraintes au début de la vidange. Contrairement à l'écoulement en masse cet écoulement (en cheminée), ne provoque pas de grandes pressions à la vidange.

\subsection{Détermination du coefficient de pression $\lambda$}

Le coefficient de pression constitue un paramètre important pour le calcul analytique des pressions dans un silo et également pour la classification des matériaux ensilés employée dans les normes. A partir des mesures de l'effort tangentiel et normal à la paroi, nous avons calculé le coefficient $\lambda$ en remplissage et en vidange centrée. Les valeurs de $\lambda$ sont consignées dans les tableaux 1 et 2 .

La figure 10, établit qu'au remplissage, le coefficient $\lambda$ reste pratiquement constant et qu'à la vidange il varie avec la profondeur. Cette variation se fait notamment sentir dans la partie supérieure de la cellule.

A la vidange, la forme des courbes de $\lambda$ en fonction de la profondeur, traduit à notre sens la forme de l'écoulement. Les fortes valeurs de $\lambda$ correspondent exactement à la zone où la matière est en mouvement et les faibles valeurs de $\lambda$ correspondent à la zone morte où la matière est en équilibre statique.

\subsection{Etat de contrainte au niveau de la paroi}

\subsubsection{Coefficient de frottement en paroi}

Le coefficient de frottement en paroi $\mu$ est déterminé directement à partir des mesures, en faisant le rapport de la contrainte $\tau$ appelée frottement et de la contrainte normale à la paroi.

Les valeurs obtenues suivant les mesures des génératrices (1) et (3) sont consignées dans les tableaux ci-dessous :

\subsubsection{Cercle de Mohr de contrainte en paroi}

Il est possible avec les résultats des capteurs de paroi de déterminer l'état de contrainte sur les éléments de matière ensilée en contact avec les parois de la cellule. Pour cela il suffit de porter sur un diagramme les mesures où les valeurs de contraintes horizontales (normales à la paroi) figurent en abscisse et celles du frottement (contrainte tangentielle) en ordonnée. On montre que pour chaque capteur les points représentatifs se trouvent sur une droite de pente $A$ et 


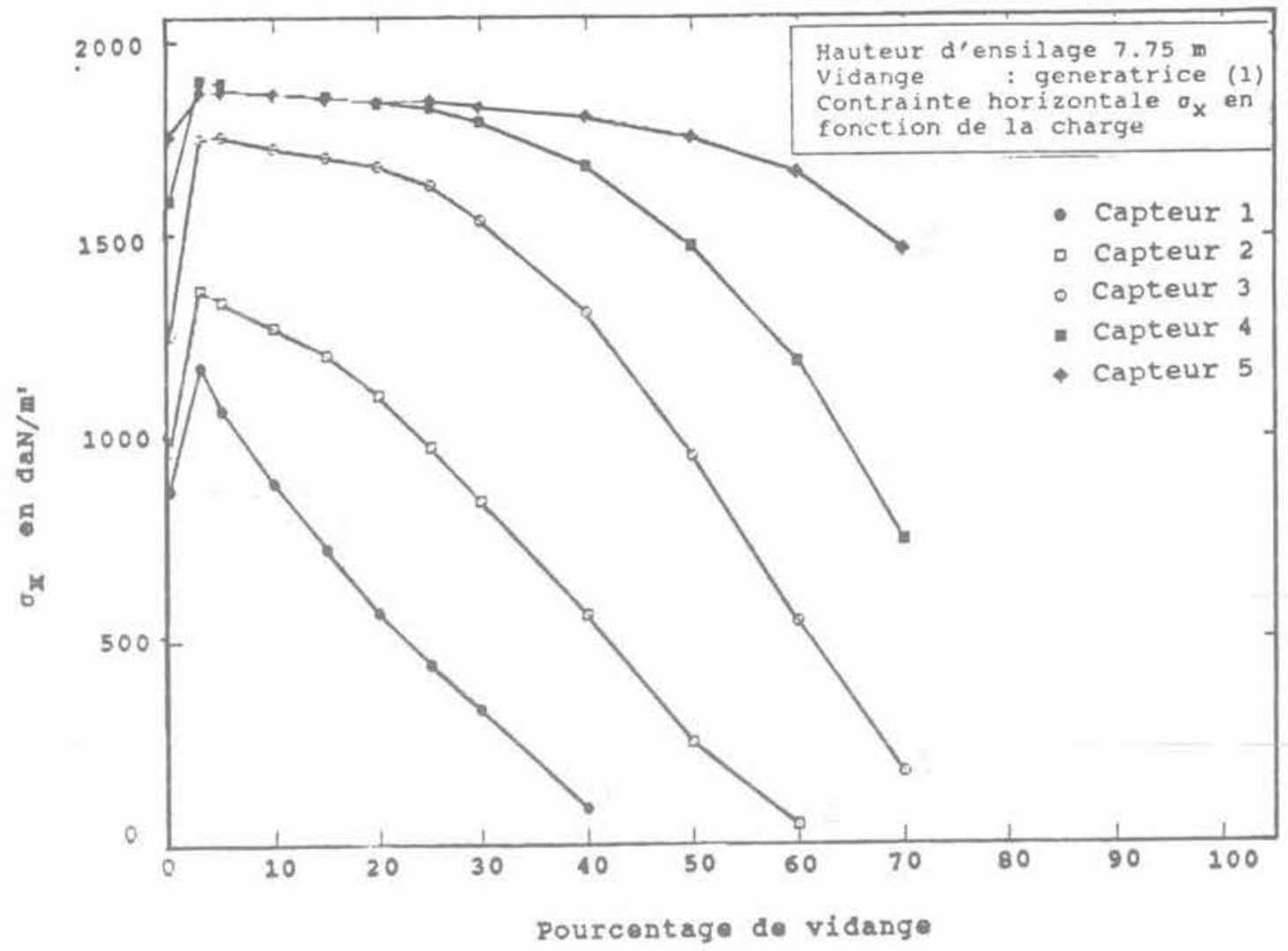

Fig. 8. - Variation de ox durant la vidange.

Fig. 8. - Variation of ox during discharge.

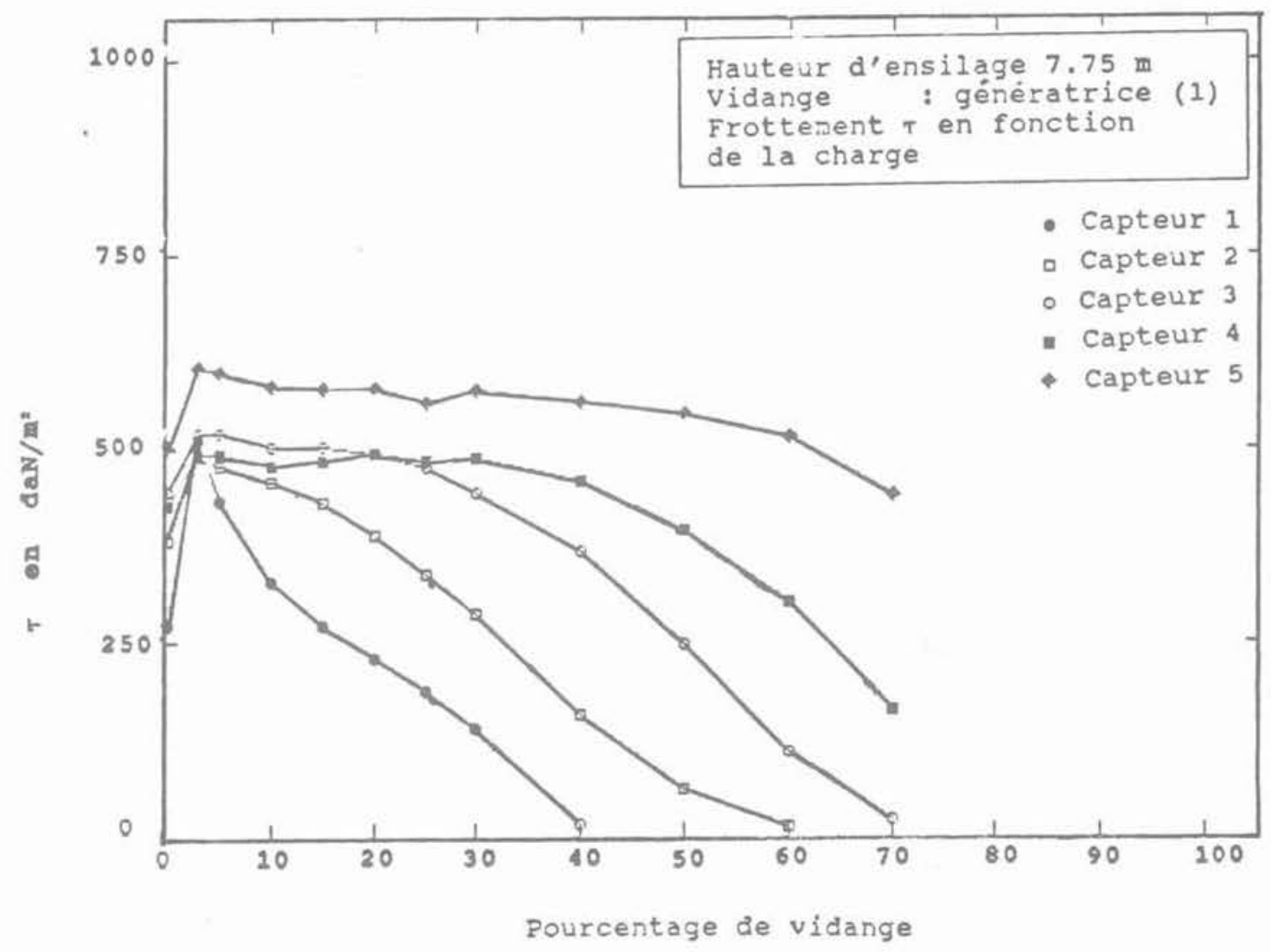

Fig. 9. - Variation de $\tau$ durant la vidange.

Fig. 9. - Variation of $\tau$ during discharge. 
Tableau 1 - Valeurs de $\lambda$ en remplissage et vidange centrée. (génératrice (1))

Table 1 - Values of $\lambda$ during filling and centric discharge. (generatrix (1))

\begin{tabular}{|c|c|c|}
\hline \multicolumn{3}{|c|}{ Génératrice (1) } \\
\hline Niveau en $\mathrm{m}$ & Remplissage & Vidange \\
\hline 0,00 & 0,362 & 0,448 \\
\hline 0,651 & 0,386 & 0,482 \\
\hline 1,767 & 0,436 & 0,582 \\
\hline 2,883 & 0,395 & 0,608 \\
\hline 3,999 & 0,431 & 0,597 \\
\hline 5,115 & 0,420 & 0,652 \\
\hline$\lambda$ moyen & 0,405 & 0,561 \\
\hline
\end{tabular}

Tableau 2 - Valeurs de $\lambda$ en remplissage et vidange centrée. (génératrice (3))

Table 2 - Values of $\lambda$ during filling and centric discharge. (generatrix (3))

\begin{tabular}{|c|c|c|}
\hline \multicolumn{3}{|c|}{ Génératrice (3) } \\
\hline Niveau en $\mathrm{m}$ & Remplissage & Vidange \\
\hline 0,00 & 0,359 & 0,406 \\
\hline 0,465 & 0,375 & 0,426 \\
\hline 1,581 & 0,415 & 0,484 \\
\hline 2,697 & 0,396 & 0,468 \\
\hline 3,813 & 0,420 & 0,585 \\
\hline 5,929 & 0,426 & 0,611 \\
\hline$\lambda$ moyen & 0,371 & 0,496 \\
\hline
\end{tabular}

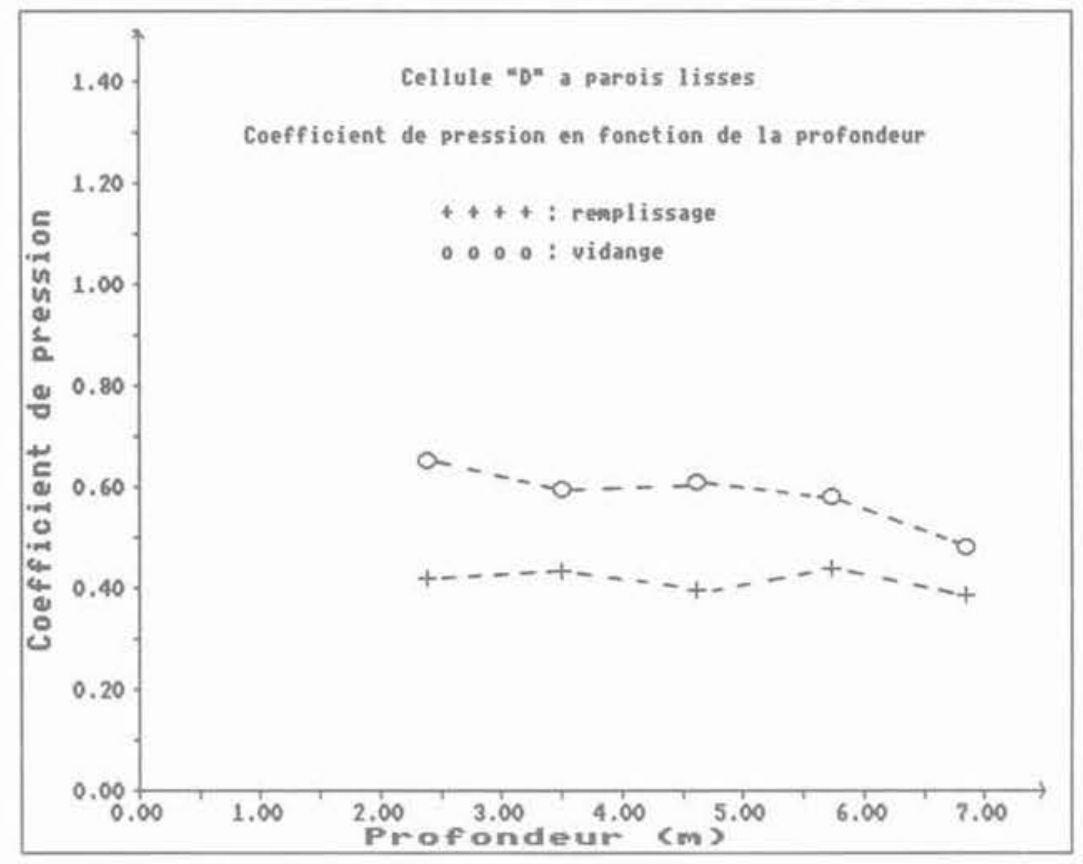

Fig. 10. - Coefficient de pression en fonction de la profondeur.

Fig. 10. - Pressure coefficent along the wall.

d'ordonnée à l'origine $\mathrm{B}$. Ceci apparaît sur la figure 11 pour le seul cas du remplissage. La contrainte de frottement qui s'exerce sur la facette d'un élément de matière s'écrit :

$$
\begin{aligned}
& \tau=\mathrm{A} \sigma \mathrm{x}+\mathrm{B} \\
& \tau=\sigma \mathrm{x} \operatorname{tg} \delta+\mathrm{c}
\end{aligned}
$$

La pente A représente le coefficient de frottement en paroi et l'ordonnée à l'origine B représente la cohésion.
Si en remplissage la loi $\tau=\sigma_{\mathrm{x}} \operatorname{tg} \delta+c$ semble être respectée, la représentation des cercles de Mohr de contraintes (fig. 12) montre la possibilité de détermination des contraintes principales au niveau de la paroi. Par contre à la vidange, il n'est pas possible d'identifier le terme de cohésion et de faire la représentation $\tau=\sigma \mathrm{x} \operatorname{tg} \delta+c$. Ceci montre la difficulté de mesure du coefficient de frottement en paroi durant la vidange. 
Tableau 3 - Coefficient de frottement en paroi. (génératrice 1)

Table 3 - Wall friction coefficient (generatrix 1)

\begin{tabular}{|c|c|c|}
\hline \multicolumn{3}{|c|}{ Génératrice (1) } \\
\hline Niveau en $m$ & Remplissage & Vidange \\
\hline 0,651 & 0,310 & 0,318 \\
\hline 1,767 & 0,263 & 0,304 \\
\hline 2,883 & 0,309 & 0,351 \\
\hline 3,999 & 0,356 & 0,457 \\
\hline 5,115 & 0,299 & 0,390 \\
\hline$\mu$ moyen & 0,307 & 0,364 \\
\hline
\end{tabular}

Tableau 4-Coefficient de frottement en paroi. (génératrice 3)

Table 4 - Wall friction coefficient. (generatrix 3)

\begin{tabular}{|c|c|c|}
\hline \multicolumn{3}{|c|}{ Génératrice (3) } \\
\hline Niveau en $m$ & Remplissage & Vidange \\
\hline 0,465 & 0,308 & 0,301 \\
\hline 1,581 & 0,256 & 0,273 \\
\hline 2,697 & 0,306 & 0,290 \\
\hline 3,813 & 0,374 & 0,328 \\
\hline 4,929 & 0,275 & 0,368 \\
\hline$\mu$ moyen & 0,304 & 0,312 \\
\hline
\end{tabular}

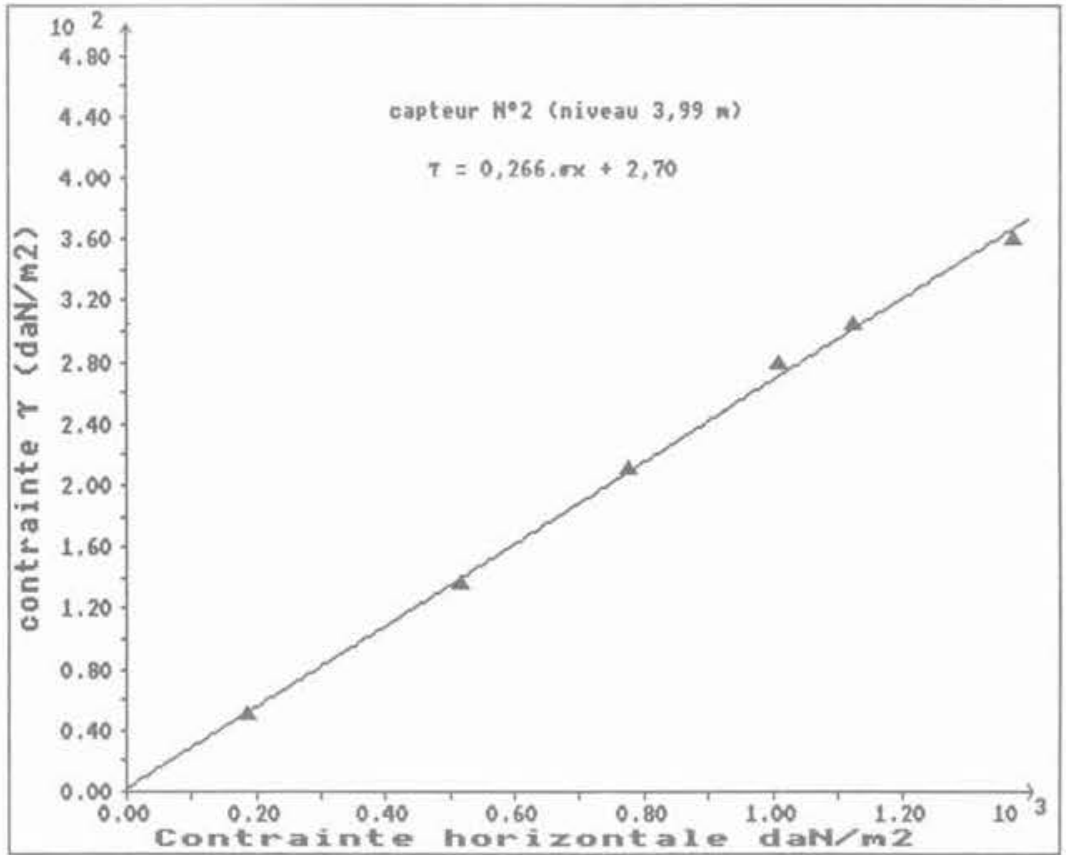

Fig. 11. - Contrainte tangentielle en fonction de la contrainte horizontale pour le capteur 2 (génératrice 1). Fig. 11. - Plot of tangential stress against normal stress for transducer 2 (generatrix 1 ).

\section{COMPARAISON ENTRE RÉSULTATS DE MESURES ET DE CALCULS}

\subsection{Remplissage}

Les courbes des figures 13 à 15 donnent la répartition de la contrainte horizontale, verticale et de frottement, le long de la paroi suivant les différentes méthodes (SOLOK (CTICM), WALTERS, norme DIN 64 et DIN 86). Nous avons porté sur ces figures les valeurs moyennes des 13 mesures obtenues à la profondeur $\mathrm{z}$ correspondant à la position de chaque capteur.
Les caractéristiques de la matière ensilée considérée sont celles recommandées par les normes dans le cas de DIN 64 et DIN 86 pour les modèles SOKOL (CTICM) et WALTERS, les valeurs suivantes sont proposées pour le blé :

$$
\begin{array}{ll}
\mu=0,327 & : \text { coefficient de frottement en paroi } \\
\varnothing=26^{\circ} & : \text { angle de frottement interne } \\
\gamma=820 \mathrm{daN} / \mathrm{m}^{3}: \text { poids volumique. }
\end{array}
$$

La comparaison des mesures avec les courbes théoriques fait apparaître une zone à la partie supérieure dans laquelle les valeurs expérimentales de contrainte 


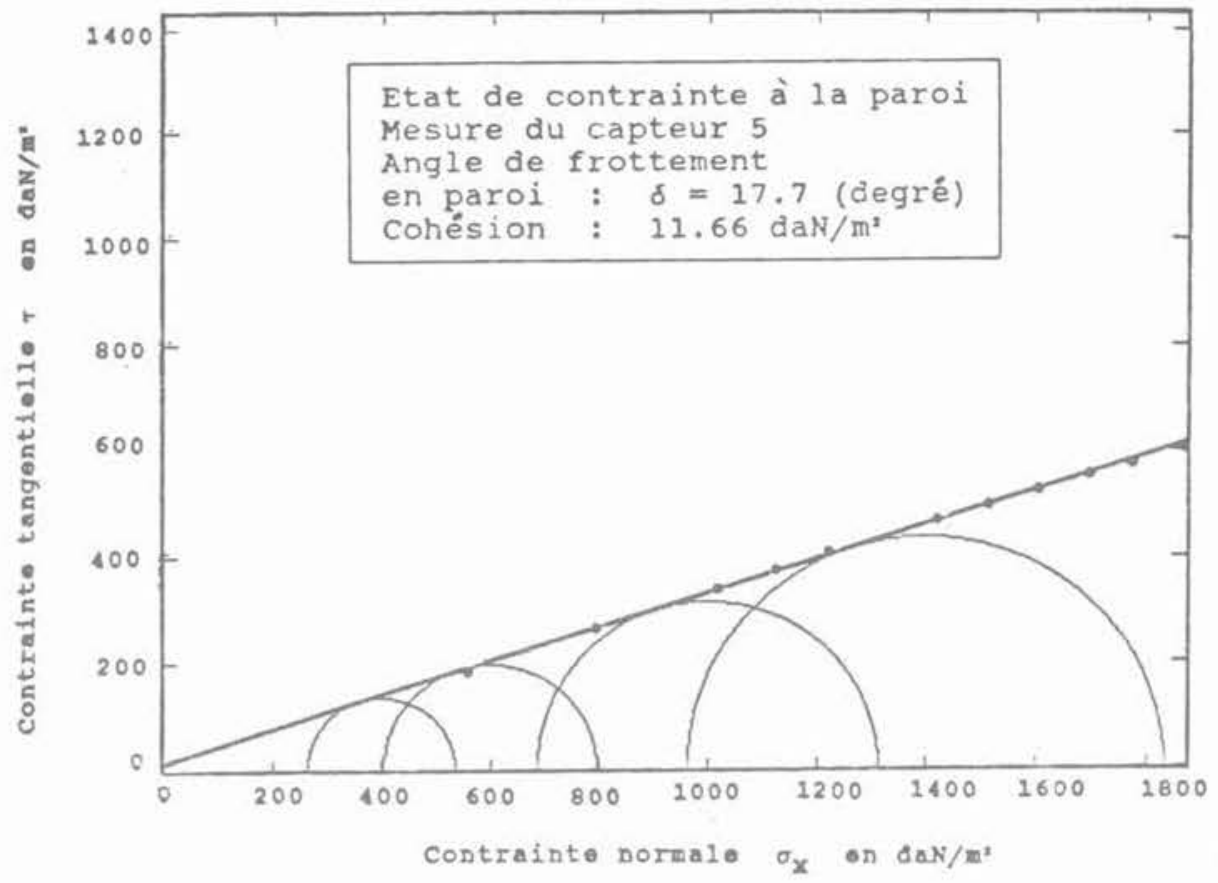

Fig. 12. - Etat de contrainte en paroi.

Fig. 12. - Wall stresses state.

horizontale et de frottement (fig. 14 et 15) dépassent légèrement les valeurs théoriques de WALTERS [15].

Les courbes de la norme DIN 64 et DIN 86 recouvrent parfaitement les résultats de mesure. II faut malgré tout remarquer que les courbes des modèles SOKOL (CTICM) et WALTERS sont comprises dans un faisceau assez resserré. La courbe de la DIN 86 s'éloigne considérablement des autres pour les 3 contraintes.

\subsection{Vidange}

Sur les figures 16 et 17 sont représentées les courbes suivant les modèles de calcul et les points expérimentaux donnant la valeur moyenne des mesures à partir des 5 essais de vidange centrée.

Pour les contraines horizontales (fig. 16), à l'exception de la norme DIN 64 pour laquelle la géométrie de la cellule est favorable, toutes les autres s'éloignent considérablement des points expérimentaux. La courbe du modèle CTICM semble toutefois plus proche des points expérimentaux que la norme DIN 86 et le modèle de WALTERS. Pour la courbe de frottement (fig. 17), on constate comme au remplissage, une zone en partie supérieure du silo dans laquelle les valeurs expérimentales de frottement sont plus élevées que celles définies par le modèle de WALTERS. Par contre dans le bas de la cellule, les valeurs expérimentales sont un peu faibles et par conséquent, recouvertes par toutes les courbes théoriques. La courbe du modèle CTICM semble la plus proche des mesures. Signalons toutefois que la DIN 86 donne des valeurs assez élevées.
A partir de ces résultats nous pouvons affirmer que les modèles théoriques et les normes applicables pour les silos dont l'écoulement s'effectue en masse, ne sont pas valables pour les cellules dont la vidange s'effectue en cheminée.

\section{CONCLUSION}

L'ensemble des résultats obtenus dans cet article concernant la loi de variation des contraintes sur les parois montre que les modèles de calcul appliqués jusqu'à présent, dans le cas de vidange en masse ne peuvent être transposés directement sur la cellule $\alpha \mathrm{D}$, type de cellule à faible élancement. En effet la vidange s'effectue en cheminée et la matière entourant l'écoulement absorbe une partie des efforts qui ne sont donc pas transmis en parois.

Du point de vue expérimental il faut signaler la qualité exceptionnelle de l'outil de travail constitué par la base expérimentale de Chartres. Cette installation sûrement unique au monde, permet grâce à une parfaite méthodologie aujourd'hui entièrement maîtrisée, d'obtenir des résultats fiables permettant une exploitation scientifique la plus complète possible.

Si la cinématique de l'écoulement n'est pas encore totalement analysable sur place il faut espérer dans un proche avenir y parvenir afin :

- de mieux maitriser les relations entre contraintes et écoulements ;

- de juger le rôle du phénomène de ségrégation des particules en cours de manipulations. 


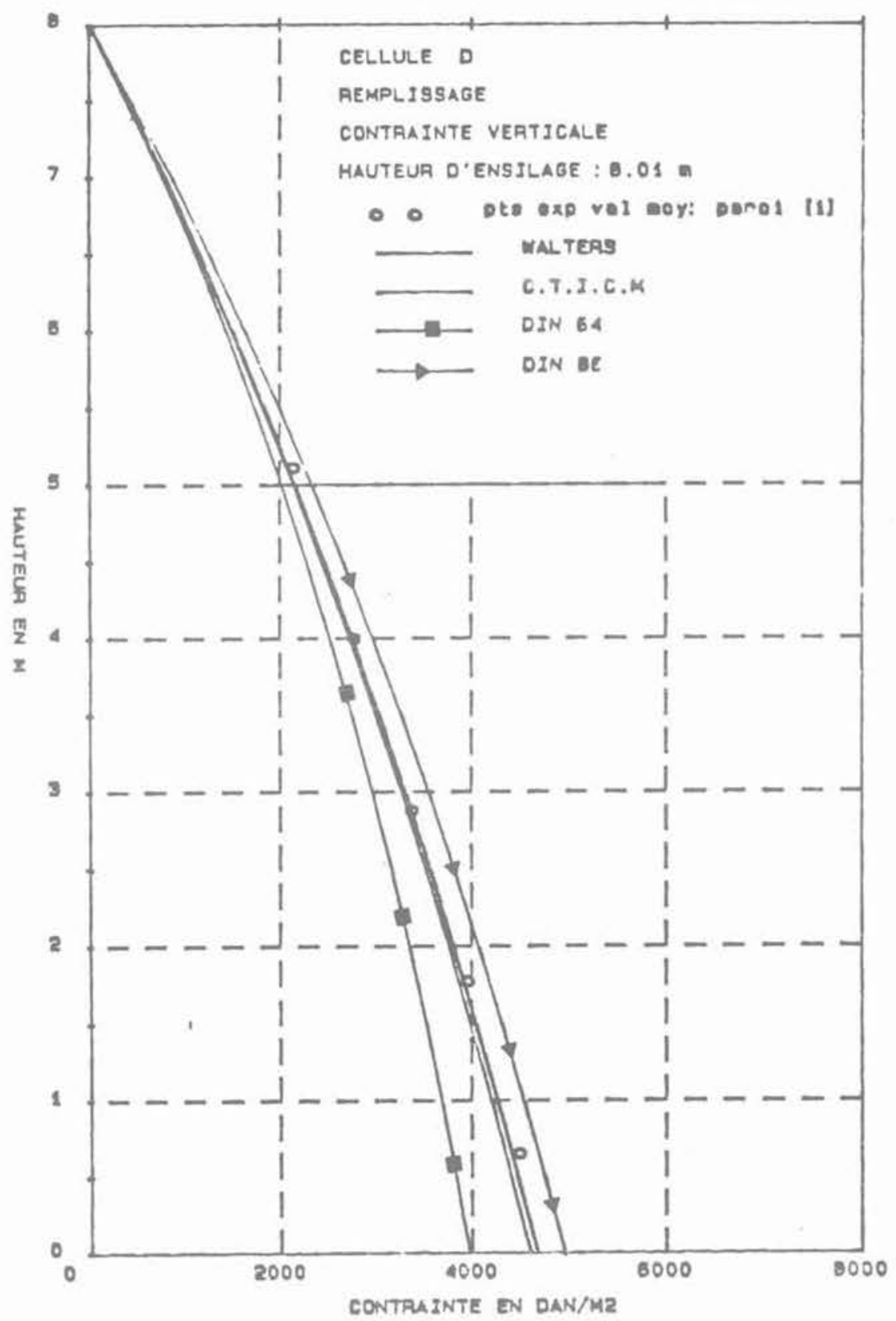

Fig. 13.

Fig. 13

\section{BIBLIOGRAPHIE}

[1] BROZZETTI J. (1989), Description de la base expérimentale d'essais de cellules métalliques de stockage. Présentation du programme d'essais. Revue CTICM n², 1989, pp. 7-17.

[2] DIN 1055 teil 6 (1964), Deutschenorm. Lastan. nahmen fur Bauten Lasten in Silozellen. Beuth verlag Gmbh Berlin 30.

[3] DIN 1055 teil 6 (1986), Deutschenorm. Lastannahmen fur Bauten Lasten in Silozellen. Beuth verlag Gmbh Berlin 30.
[4] FREESE B. (1977), Druckuerthaltnisse in zylindrischen Silozelen. Dissertation Universität Karlsruhe, Tag der mündlichen Prüfung 25.02.1977.

[5] GAYLORD E.H. et G.H. (1984), Design of steel bins for storage of bulk solids, Prentice-Hall, INC., Englewood Cliffs, New Jersey.

[6] JENIKE A.W., JOHANSON J.R. (1968), Bin loads. Journal of structural division St4, pp. 1011-1037.

[7] JENIKE A.W., JOHANSON J.R., CARSON J.W. (1972), Bin loads part 2/3. Concepts Journal of Engng JOHANSON and for industry Feb. 1972. 


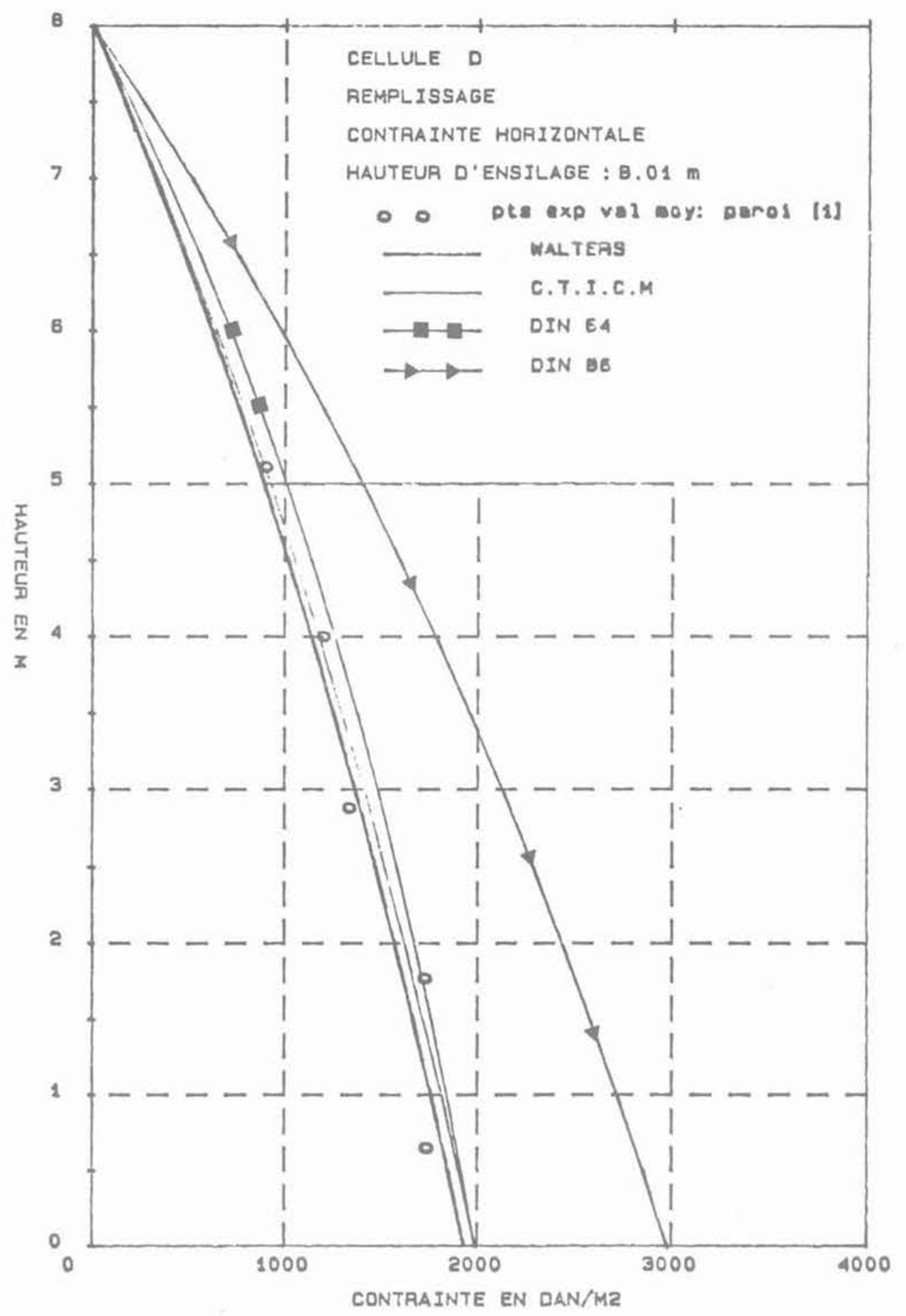

Fig. 14.

Fig. 14. 


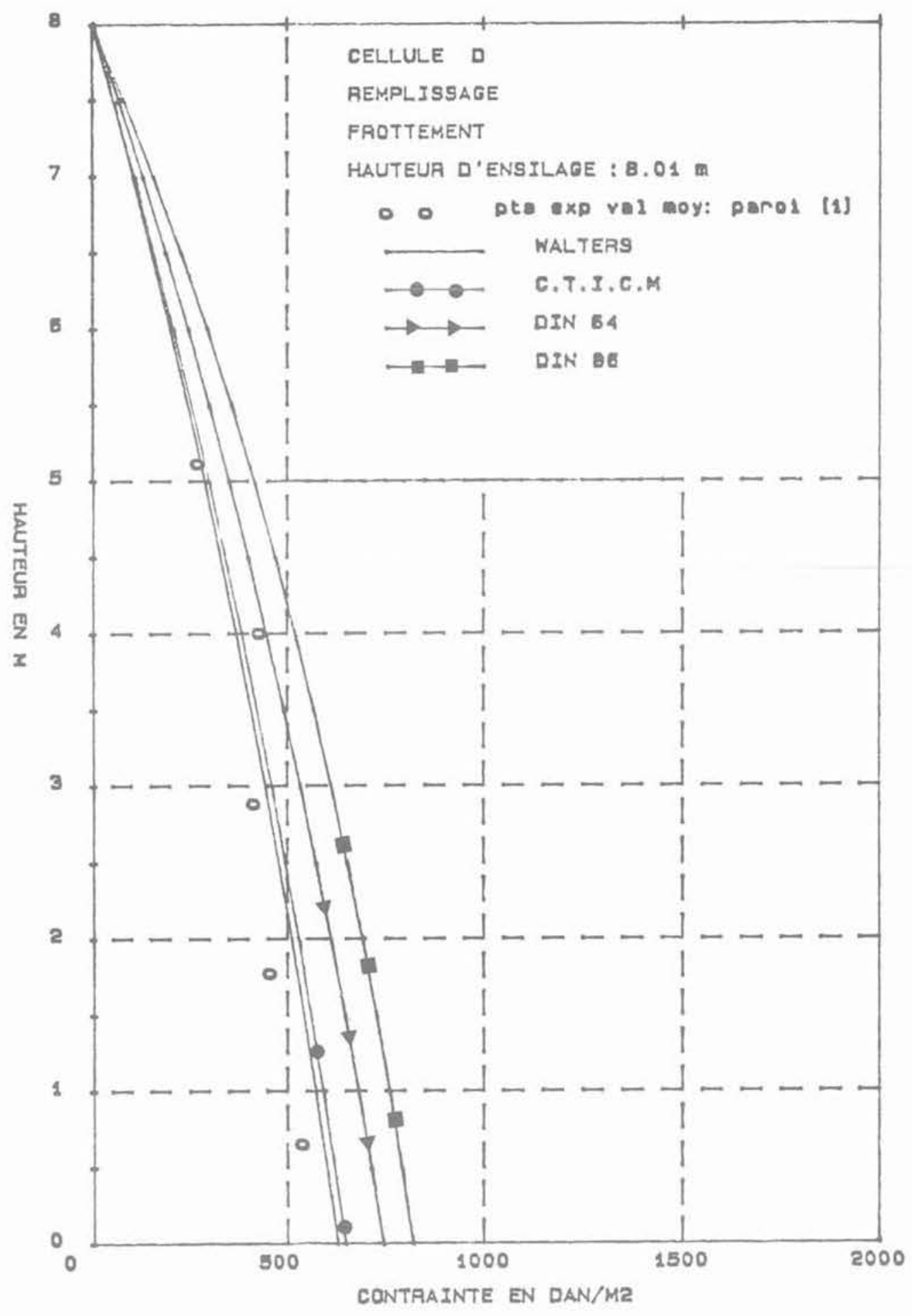

Fig. 15.

Fig. 15. 


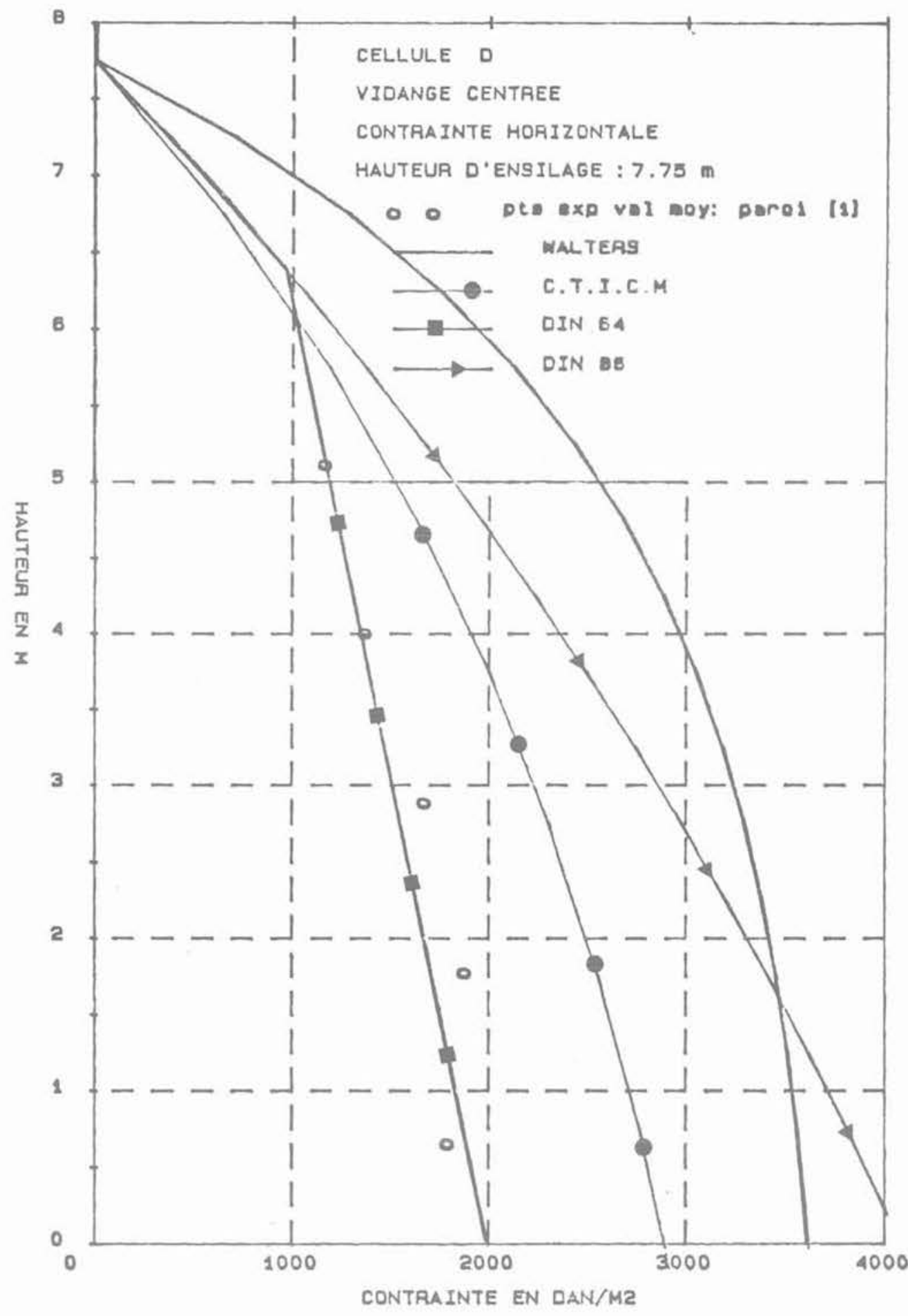

Fig. 16.

Fig. 16. 


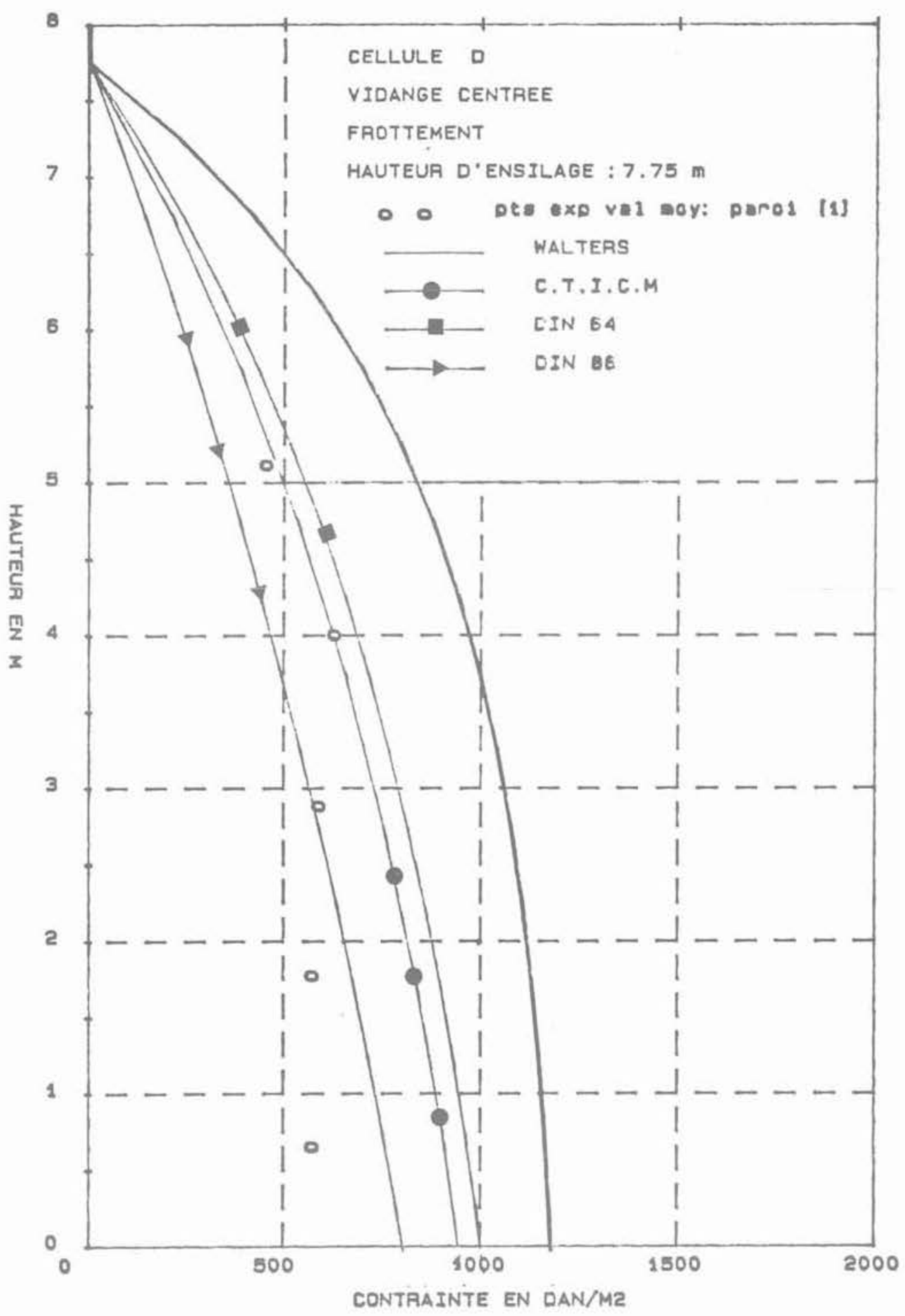

Fig. 17.

Fig. 17 
[8] KHELIL A. (1989), Etude du champ de vitesses et de contraintes dans les silos métalliques. Thèse présentée à l'INPL de Nancy, soutenue le 3 février 1989, pour obtenir le grade de Docteur de l'INPL.

[9] KOLYMBAS D. (1987), A constitutive law of the rate type for soils and other granular materials. Proceedings Numerical methods in geomechanics, Kosice, May 1987.

[10] LUMBROSO A. (1977), Bases scientifiques du calcul des enceintes renfermant des massifs pulvérulents et du calcul des silos. Annales de IITBTP, série TMC/202, janvier 1977.

[11] LADE P.V. (1977), Elasto-plastique stress-strain theory for cohesionless soil with curved surfaces, International Journal of Solids structures, vol. 13 (1978), pp. 1019-1035.

[12] REIMBERT M. et A. (1982), Silos, théorie et pratique. Edition Eyrolles, 1982
[13] SAFARIAN S., HARRIS C. (1985), Design and construction of silos and bunkers, Van Nostrand Reinhold 1985.

[14] SOKOL L. (1984), Bin loads in vertical silos. International journal of bulk solids in silos, vol. $1, n^{\circ} 1,1984$

[15] WALTERS J.K. (1973), A theoritical analysis of stresses in silos with vertical walls. Chemical Engng. Sc, vol. 21, 1973, pp. 13-21.

\section{REMERCIEMENTS}

Cette étude s'inscrit dans l'ensemble des travaux entrepris par le Ministère de la Recherche et de la Technologie en liaison avec le CTICM et les organismes professionnels des industries céréalières et de la construction métallique. 